\title{
Contribuições das equipes de Consultório na Rua para o cuidado e a gestão da atenção básica
}

\author{
Everson Rach Vargas $^{1}$ e lacã Macerata ${ }^{1}$
}

Como citar Vargas ER, Macerata I. Contribuições das equipes de Consultório na Rua para o cuidado e a gestão da atenção básica. Rev Panam Salud Publica. 2018;42:e170. https://doi.org/10.26633/RPSP.2018.170

RESUMO Como parte do Sistema Único de Saúde (SUS) no Brasil, os Consultórios na Rua e suas equipes foram criados tendo como função prioritária o desenvolvimento de cuidados primários e a garantia de acesso às ações e serviços de saúde para populações em situação de rua no próprio ambiente da rua, criando vínculos dessa população com outros serviços que não sejam somente de urgência e emergência. Seu escopo de atividades envolve, além da atenção, a proteção contra os riscos a que essa população está exposta, combinada com a busca da garantia de seus direitos. Nesse sentido, os Consultórios na Rua buscam efetivar a equidade e o acesso a ações e serviços de saúde para uma população sem domicílio fixo dentro de um sistema baseado essencialmente na adscrição territorial da população. Assim, a criação do Consultório na Rua inaugura novos modos de cuidar em saúde e, consequentemente, novos modos de fazer a gestão do processo de trabalho. A partir dessa articulação entre cuidado e gestão, o presente artigo discute três planos de intervenção onde se dá a prática das equipes de Consultório na Rua - a própria rua, a sede/unidade de referência e as redes institucionais -, sua relação com os demais serviços de atenção primária à saúde (APS) e a sua contribuição para reconciliar a APS com os seus atributos fundamentais, para além da adscrição do território geográfico.

Palavras-chave Gestão em saúde; acesso universal aos serviços de saúde; atenção primária à saúde; pessoas em situação de rua; Brasil.

Desde a Declaração de Alma-Ata, em 1978 (1), a atenção primária à saúde (APS) se consolidou como serviço preferencial e porta de entrada dos sistemas de saúde. A estruturação desse nível de atenção reforça a importância da atenção primária em um contexto de saúde historicamente constituído pela atuação em

Universidade Federal Fluminense (UFF), Programa de Pós-Graduação em Psicologia, Rio de Janeiro (RJ), Brasil. Correspondência: Everson Rach Vargas, eversonpsi@gmail.com cuidados de alta densidade tecnológica e no tratamento de agravos previamente instalados.

No Brasil, as equipes de Consultório na Rua (eCR) foram instituídas a partir da segunda edição da Política Nacional de Atenção Básica (PNAB), de 2011. Tratam-se de equipes itinerantes que atuam, privilegiadamente, em pontos de maior concentração de pessoas em situação de rua. As ações dessas equipes se diversificam de acordo com as necessidades de saúde identificadas, das mais pontuais às crônicas, situações essas que acabam por demandar um acompanhamento longitudinal.

As eCR têm como função prioritária o desenvolvimento de cuidados primários, prestando assistência voltada aos agravos mais prevalentes das pessoas em situação de rua, com distribuição de insumos e orientações em saúde, e garantindo o acesso às ações e serviços a partir da própria rua, criando vínculos dessa população com outros serviços que não sejam somente de urgência e 
emergência (2). Além disso, seu escopo de atividades envolve a proteção contra os risco a que essa população está exposta, combinada com a busca da garantia de seus direitos.

Os Consultórios na Rua surgiram em um contexto de ampliação dos direitos sociais no Brasil pela extensão de políticas públicas a populações que antes não tinham acesso a direitos básicos garantidos pela Constituição brasileira, de forma a intervir nos coeficientes de desigualdade social. Também contribuíram para o seu surgimento a aposta, por parte do Ministério da Saúde, na APS como ordenadora do sistema e a priorização das redes de atenção à saúde (RAS); o controverso e questionável plano de enfrentamento ao crack, surgido no bojo dos megaeventos realizados nos anos 2010 no Rio de Janeiro (Copa do Mundo da FIFA e Olimpíadas) (3); e a crescente organização e participação do Movimento Nacional da População em Situação de Rua na arena política das grandes cidades brasileiras.

A criação das eCR se justifica pela grande vulnerabilidade das populações em situação de rua, juntamente com a baixa capacidade da rede de atenção básica (como se denomina a APS no Brasil) existente de acolhê-las. Esse fato reflete uma infinidade de dificuldades relacionadas ao modelo de APS adotado no Brasil, que, em linhas gerais, inclui a organização a partir de domicílio fixo para a definição de um território adscrito. Esse modelo impede o acompanhamento da dinâmica da rua e impõe uma barreira histórica no acesso dessas pessoas aos serviços de saúde.

O território de vida da população em situação de rua coloca uma série de desafios ao SUS no que diz respeito à concretização de seus princípios, entre os quais a construção da integralidade do cuidado, ou seja, o desenvolvimento de um olhar mais integral e menos "especialista" nas práticas de cuidado. Isso se desdobra em duas questões: na maneira como geralmente se considera o território - apenas como demarcação geográfica ou matriz do domicílio - de forma que só fariam parte desse território aqueles oficialmente domiciliados nele; e na maneira como os diferentes saberes de uma equipe multiprofissional compõem uma prática de atenção, de modo que as dimensões biopsicossocias não sejam desmembradas (4). Junto à população em situação de rua, os conceitos que organizam a APS são colocados em xeque: nem todos os habitantes de um território são domiciliados; e os processos de saúde-doença biológicos, subjetivos e sociais estão articulados e mutuamente apoiados.

Foi para aumentar o grau de equidade do Sistema que o Consultório na Rua se institucionalizou. A vulnerabilidade dessa população evidencia as vulnerabilidades das práticas de cuidado do SUS, mas também mostra que o trabalho das eCR traz uma série de inovações para a prática de cuidado na APS, bem como para a gestão do processo de trabalho.

A partir disso, o presente texto é uma reflexão construída com base em uma série de experiências, em diferentes campos, com o Consultório na Rua: experiência de trabalho vivida na construção inicial de um desses dispositivos, na cidade do Rio de Janeiro; experiência de pesquisa de doutorado acerca da prática de cuidado realizada por essa mesma equipe, a qual gerou um documento técnico sobre a prática; e experiência de construção de material didático para um curso de formação de profissionais para o Consultório na Rua (5).

A partir de questões suscitadas pela implementação dessas equipes na APS brasileira, pretende-se traçar um panorama de como as eCR contribuem para reconciliar os demais serviços de APS com suas diretrizes e sua missão. Esta análise foca, especificamente, em como o modo de realizar a gestão do processo de trabalho da eCR está extremamente articulado com as práticas de cuidado, não havendo, portanto, separação entre gestão e atenção em saúde. Essa articulação promovida pelas eCR pode, enfim, apontar alguns problemas que são re-apresentados para os demais serviços de APS, como, por exemplo, as equipes de Saúde da Família.

\section{GESTÃO E CUIDADO EM SAÚDE: O PROCESSO DE TRABALHO}

A gestão, originada no campo da administração, é pensada como atividade que tem a função de exercer um mandato sobre o trabalho e qualificar seus processos. Na modernidade, a gestão ganhou centralidade na produção industrial, sendo caracterizada, nos exemplos de Taylor e Ford, por racionalização, divisão e mecanização dos processos de trabalho, que deveriam ser centralmente controlados em seus mínimos gestos. O campo da saúde coletiva tomou como problema a gestão taylorista (6), apostando que a alteração dos modelos de atenção passava pela alteração dos modelos de gestão: desenvolver outra racionalidade para os processos de gestão, diferente da administrativa ou privada.

Assim, há uma especificidade da gestão no campo da saúde: a gestão do processo de trabalho e as práticas de cuidado, embora sejam dimensões distintas, são inseparáveis (7). As práticas de cuidado são coproduzidas com as práticas de gestão. Em toda prática de gestão, há uma dimensão de cuidado, e em toda prática de cuidado, há uma dimensão de gestão. A especificidade dos processos de gestão na área da saúde é que eles devem estar integrados com a produção do cuidado. Produzir cuidado é construir uma prática a partir das especificidades do objeto de cuidado: pessoas e territórios singulares. Contudo, a produção do cuidado envolve um certo modo de produzir gestão, ou seja, o gerir o processo de trabalho em saúde é fazer a gestão do cuidado.

Essa gestão do trabalho em saúde irá se ocupar com a coordenação do trabalho, envolvendo os diversos atores implicados em seu território de práticas - trabalhadores, usuários, comunidade. Daí o sentido reafirmado da cogestão (7). Assim, na diretriz de uma cogestão, gerir não significa colocar-se sobre o trabalho e o território onde ele se desenvolve - perspectiva clássica - a fim de regulamentá-los. Significa, isso sim, posicionar-se com o trabalho e o território, fazendo composição com e a partir deles. Nesse sentido de gestão, o cuidado não é pensado como uma ação sobre o outro - concepção hierarquizada, verticalizada e centralizada, taylorista. Escapando da função tutelar, classicamente atribuída ao cuidar, a efetivação do cuidado não depende somente da técnica ou da ação central do trabalhador, mas envolve os vários atores, recursos e dinâmicas do território de ação. Território, aqui, tem o sentido de categoria que define relações complexas e situadas entre diversos vetores, e que envolve dimensões objetivas e subjetivas (4). E é nessa relação com, no e a partir do território, e não sobre ou contra ele, que o cuidado se faz como prática coletiva, democrática e inclusiva, seguindo a diretriz de uma clínica ampliada $(4,6,7)$. 
Em um cuidado verticalizado e centralizado em procedimentos, saúde e usuário se tornam categorias universais e não situadas, e o cuidado assume uma perigosa função de gestão calculada da vida (8). Cuidado e gestão do processo de cuidado se fazem no encontro com sujeitos e territórios, sendo esse encontro um dos momentos mais singulares do processo de trabalho em saúde (9). O modo de gestão desse trabalho deve se apropriar dessa mesma perspectiva: uma gestão com; uma cogestão. Cogestão é uma diretriz. Perpassa os processos no campo da saúde em maior ou menor grau, mas sempre está, em alguma medida, presente. A gestão não se limita ao ato do gestor, mas está nos atos clínicos e em todos os âmbitos dos serviços e do processo de trabalho.

Na perspectiva da cogestão, o cuidado e a política também são inseparáveis $(4,10)$ : a gestão assume e cria condições para o cuidado. Ele é necessariamente político, na medida em que está sempre inserido em um campo de disputas, de relações de poder, podendo produzir práticas tanto de dominação quanto de liberação e produção de autonomia. Há sempre o risco de se produzir, como efeitos subjetivos, o constrangimento e a padronização dos modos de viver. Essa é uma problemática que se coloca especialmente na APS, cujos serviços atuam de maneira muito próxima ao cotidiano da vida dos usuários.

Assim, a gestão do cuidado na APS tem especificidades que trazem a necessidade de deslocamentos da gestão advinda da administração privada. Especialmente na prática das eCR, tanto o cuidado como a gestão devem construir e gerir o processo de trabalho tendo como principal ponto de apoio a relação do serviço/equipe com o território.

\section{PLANOS DE INTERVENÇÃO DAS EQUIPES DE CONSULTÓRIO NA RUA}

A prática do Consultório na Rua, com sua aposta em uma proximidade e atuação no território e a partir de suas singularidades, mostra que o processo de trabalho se dá através de subdivisões, a partir de planos de intervenção das eCR. Por sua vez, esses planos exigem gestão dos processos de trabalho mais específicos.

O Consultório na Rua tem três planos fundamentais de atuação: a rua; a sede/ unidade de referência; e as redes institucionais (saúde e intersetoriais). Por esses planos perpassam toda a gestão e a produção do cuidado. A noção de planos ultrapassa a ideia de espaço como espaço físico euclidiano, fixo, imutável, com clara demarcação, visível, com fronteiras bem definidas. Compreendemos que tais planos estão presentes uns nos outros, tornando possível identificar influências e modos de atuar que um plano exerce sobre os outros. Assim, o plano afirma a dependência entre os diferentes espaços do trabalho, já que eles não se estabeleçam como unidades estáveis.

$\mathrm{Na}$ perspectiva transdisciplinar (11), compreendem-se os espaços de trabalho como porosos à multiplicidade de fluxos que os atravessam. Por constituírem-se de aspectos objetivos e subjetivos, esses planos se interpenetram, se misturam. Há um pouco da rua na sede/unidade de referência e há um pouco da sede/ unidade de referência na rua, por exemplo. A rua como plano denota não só o seu sentido objetivo, como demarcação física, mas também subjetivo - certas qualidades da rua em meio à sede/unidade de referência.

O plano de intervenção "rua" é o território de vida das pessoas que são cuidadas. Esse território é muito diferente dos territórios de vida mais comuns em uma cidade. É território de moradia e de vida sem ser domiciliar. Entendido não somente por sua organização física e geográfica, mas também por sua organização combinada a um funcionamento subjetivo, caracterizando-se tanto por aspectos visíveis quanto por aspectos invisíveis.

Os aspectos visíveis da rua são os que podem ser mapeados e sistematizados: o número de pessoas, a delimitação territorial, os dados epidemiológicos que subsidiam a avaliação e o monitoramento das práticas, as situações concretas de violência, os recursos, o tamanho, a movimentação, entre muitos outros. O território adscrito de uma equipe é composto, então, por tipos de população em situação de rua (mais fixas, mais itinerantes), atores variados que se fazem presentes (moradores de rua, comerciantes, polícia, agentes de outras políticas públicas, transeuntes, traficantes, grupos de extermínio, instituições religiosas, etc.), recursos que o território oferece à população em situação de rua (comida, dinheiro, local para dormir, outros serviços assistenciais) e tipos de agravos em saúde mais prevalentes, entre outros.
Os aspectos invisíveis da rua apresentam-se como dinâmicas relacionais que usuários ou grupos estabelecem num determinado território. Essa dimensão subjetiva da rua fala das relações que se estabelecem nela, do lugar que ela ocupa na vida dos sujeitos (lugares afetivos e simbólicos). Apesar de esses aspectos não serem palpáveis, eles existem, e na hora de organizar o processo de trabalho é preciso considerá-los. Isso cria a necessidade de repensar e reconsiderar as estratégias e os conceitos de saúde e, ainda, o engajamento no território. Deve-se entender o território, senti-lo; enxergá-lo e respirar seus ares.

A "sede/unidade de referência" é tradicionalmente compreendida como local onde se organizam e se dão as práticas de cuidado. Como plano de intervenção do Consultório na Rua, a sede se faz presente também na rua e na rede, indo além das práticas propriamente realizadas e previstas na unidade de saúde. O principal fator que define um espaço de cuidado é o aspecto relacional (12): a maneira de organizar e operar os processos e os fluxos de trabalho na sua relação com a realidade do território e do sujeito, com seus modos de viver. Dessa forma, um espaço de cuidado é realizado através de um modo de entrar em relação, para além da estrutura e limites do serviço no qual estão lotados os trabalhadores, ou até mesmo para além dos procedimentos tradicionais, rapidamente classificados como cuidado. Podem ser constituídos espaços de cuidado na rua da mesma forma que podemos ter uma sede ou um equipamento da rede que, embora tenha o mandato do cuidado, efetivamente não cuida.

O aspecto relacional do espaço de cuidado é percebido quando o Consultório na Rua torna-se referência no território, seja na sua presença na rua, seja na sede ou na rede. De uma maneira ou de outra, a sede se faz presente na rua e a rua se faz presente na sede. É preciso organizar o espaço da sede como uma espaço de acolhimento para os usuários, onde os fluxos do espaço se adequem à rua: flexibilização e alteração dos horários de funcionamento, dos documentos exigidos para atendimento, dos modos de ouvir, falar e orientar, enfim, acolher. Em nossa experiência isso exigiu, por exemplo, ter sempre um trabalhador do Consultório na Rua na sede, para facilitar as relações entre os usuários do Consultório 
na Rua e os profissionais e usuários de outros programas; exigiu manter uma sala de espera que considerasse as urgências não só do corpo biológico, mas também subjetivas e sociais - como por exemplo alguém que precisaria ir a uma entrevista de emprego, ou alguém que estava desaparecido há muito tempo, ou que estava em crise de abstinência de drogas.

No contexto de uma política de saúde voltada para uma população historicamente à margem dos serviços de saúde, é importante considerar que as inúmeras situações graves apresentadas por essa população, associadas à atual fragmentação do trabalho em rede e à insuficiência de retaguarda clínica na atenção secundária e terciária, produzem a necessidade de o Consultório na Rua dispor de um espaço para resolutividade de questões específicas: curativos, exame citopatológico. Mesmo sendo itinerante, a equipe precisa ter uma sede de referência, que tenha porta aberta à população em situação de rua, que possa ser utilizada sem restrições, sem necessidade de nenhum tipo de autorização, um espaço onde a pessoa em situação de rua tenha seu direito de acesso garantido. É preciso que se crie, no plano da rua, o plano da sede: espaço de encontro e acolhimento itinerante, junto ao local onde pessoa habita. A existência da sede, com seus recursos e fluxos, não ficará restrita às paredes do serviço, mas acontece e atravessa o plano da rua e da rede e, consequentemente, o seu modo de funcionamento.

O plano de intervenção "rede" se refere aos espaços físicos (serviços) e às lógicas organizacionais dos equipamentos da saúde, de outras políticas públicas e da sociedade civil, na relação com o trabalho do Consultório na Rua. O plano rede é composto pelos seus pontos (serviços e ações específicas de saúde e de outros setores) e também pelos modos como esses serviços, trabalhadores e usuários entram em relação.

A recente construção das redes de atenção à saúde (RAS) (13) realça o peculiar trabalho realizado pela APS, que tem como prerrogativa a coordenação do cuidado e a ordenação das diferentes redes em seu território. As RAS devem ter missão e objetivos comuns, operando de forma cooperativa e interdependente, trocando recursos. Não devem ser estabelecidas hierarquias entre os diferentes componentes. Todos os pontos de atenção à saúde são igualmente importantes e se relacionam horizontalmente. Devem focar-se no ciclo completo de atenção a uma condição de saúde e têm responsabilidades sanitárias e econômicas inequívocas por sua população (14). Em que pese a relevância de organização interna do seu funcionamento, o Sistema Único de Saúde (SUS) no Brasil não pode se apresentar como alternativa absoluta para atender a complexidade das demandas apresentadas pelas populações em situação de rua. Assim, a rede intersetorial também compõe esse plano de intervenção do Consultório na Rua.

A rede se faz presente na dimensão rua, por exemplo, quando algum comerciante ajuda no tratamento de um paciente, quando o Serviço de Atendimento Móvel de Urgência (SAMU) entra em ação, ou quando é necessário dialogar com a polícia, os traficantes ou os moradores da área. No plano sede, um exemplo da presença da rede seria a construção de um projeto terapêutico integrado com outro serviço. O Consultório na Rua tem a função de tecer e aquecer a rede, criando relações, negociando, entendendo a lógica e a função de cada serviço, acolhendo suas dificuldades, mas também tensionando a acolhida da rua e suas especificidades. Na construção desse plano, está intrínseca toda a perspectiva do cuidado e de organização do processo de trabalho das eCR, que aponta para a urgência em se pensar práticas e políticas que atendam à necessidade da rua. Sobretudo, pensar sobre como construir uma rede intersetorial.

Articular uma rede é fazer fluir o serviço em suas dinâmicas interna e externa. A articulação não é responsabilidade apenas dos gestores, mas se caracteriza na função-gestor, ou seja, de todos os trabalhadores e, inclusive, do usuário. Fazer fluir é estabelecer fluxos que ajudem o trabalho a se consolidar, que permitam que o acompanhamento se efetive e que desfaçam "nós" de comunicação e barreiras com o intuito de tornar o trabalho tanto mais resolutivo quanto mais for compartilhado.

As redes se constituem em arranjos entre os diferentes serviços de saúde nos territórios para desconstruir a fragmentação das práticas de saúde sustentadas apenas pela lógica de atendimentos pontuais aos agravos, geralmente centrada na lógica orgânica-biológica. Experiências demonstram que a integração sistêmica prevista pelas RAS responde de maneira mais eficaz tanto à organização interna do sistema de saúde quanto aos desafios do cenário socioeconômico, demográfico, epidemiológico e sanitário dos territórios. A constituição da rede fundamenta-se na concepção de que a APS deve resolver os problemas mais comuns de saúde, coordenando o cuidado dos usuários que estejam em todos os outros pontos de atenção (13). Sendo assim, a construção da rede é uma tarefa inseparável do cuidado e exige tempo na agenda dos trabalhadores das eCR para construí-la e para mantê-la aquecida.

\section{CONSIDERAÇÕES FINAIS}

As problemáticas e aspectos aqui brevemente levantados ganham relevo a partir de experiências concretas com o Consultório na Rua, e não dizem respeito apenas ao trabalho dessas equipes. Quando confrontamos a realidade mais ampla dos serviços de APS, vemos muitos pontos de comunicação, principalmente no que diz respeito à relação entre prática de cuidado, gestão do processo de trabalho e território. Cada vez menos o Consultório na Rua aparece como um serviço especializado. Ele figura também como um serviço analisador, ou seja, que coloca em análise as práticas em saúde.

Para além de se constituir como uma estrutura voltada para os cuidados primários, para a prevenção e a promoção, com alta capacidade de capilarizar as ações de saúde, a APS se caracteriza por ser um serviço de base territorial. Isso não significa apenas "adscrição de território", o que, em última análise, restringe toda a potencialidade desse nível de atenção. No Brasil, não é raro serviços de APS se tornarem limitados ao espaço delimitado da sede/unidade de referência, ficando alheios à dinâmica territorial. O que, por sua vez, impele sua prática a centrar-se no procedimento "queixa-conduta", descaracterizando o que queremos afirmar como cuidado. $\mathrm{O}$ modo de relação entre equipe e território aqui exposto pode fornecer elementos primordiais para as práticas de cuidado e também para as práticas de gestão do cuidado.

A gestão do processo de trabalho aqui proposta é realizada, radicalmente, a partir das demandas construídas na relação entre dinâmicas da equipe e dinâmicas do território. Construir a gestão do trabalho nessas bases - a base da 
relação -, pode permitir reconciliar o modelo de APS com a sua missão: cuidado de $80 \%$ dos agravos em saúde através de uma lógica territorial: no, através e com o território, sendo efetivamente um equipamento de ampliação de acesso. Contudo, esses modos de relação com o território permitem não somente um aumento da abrangência, mas também um aumento do grau da capacidade de cuidar de situações complexas, onde vários concorrem. Um duplo sentido de ampliação: ampliar a capacidade de acesso Declaraçãoo de Alma-Ata. Conferência Internacional sobre Cuidados Primários em Saúde. Alma-Ata; 1978. Disponível em: www.who.int/publications/almaata declaration_en.pdf?ua $=1 \& u a=1$ Acessado em setembro de 2018 .

2. Rosa AS, Cavicchioli MG, Brêtas AC. O processo saúde-doença-cuidado e a população em situação de rua. Rev Latino-Am Enfermagem. 2005;13(4):576-82.

3. Decreto 7 179/2010. Disponível em: http:/ / www.mds.gov.br/webarquivos/legislacao/assistencia_social/decreto/decreto_7179.pdf Acessado em setembro de 2018.

4. Machado IM. Traços de uma clínica de território: intervenção clínico-política na atenção básica com a rua [tese de doutorado]. Rio de Janeiro: Programa de PósGraduação em Psicologia, Universidade Federal Fluminense; 2015.

5. Lopes LE, organizador. Caderno de atividades: tutor: curso de atenção integral à saúde de pessoas em situação de rua com ênfase nas equipes de consultório na rua. Rio de Janeiro: Ensino à Distância/Escola Nacional de Saúde Pública; 2014. e atenção; ampliar a capacidade de considerar e intervir junto aos vários vetores que compõem uma situação complexa, seja de um indivíduo, grupo ou comunidade. Uma reconciliação entre APS e território, pela construção de novas práticas de atenção diretamente implicadas em novas práticas de gestão do processo de trabalho para o SUS.

Agradecimentos. À Coordenação de Aperfeiçoamento de Pessoal de Nível Superior (CAPES) pela concessão

\section{REFERÊNCIAS}

6. Sousa GC. O anti-Taylor: sobre a invenção de um método para co-governar instituições de saúde produzindo liberdade e compromisso. Cad Saude Publica. 1998;14(4): 863-70.

7. Brasil, Política Nacional de Humanização da Atenção e Gestão do SUS. Brasília: Ministério da Saúde; 2009. Disponível em: http: / /bvsms.saude.gov.br/bvs/publicacoes/gestao_participativa_cogestao.pdf Acessado em agosto de 2018.

8. Alexandre FS. A parresía pedagógica de Foucault e o êthos da educação como psicagogia. Rev Bras Educ. 2013;18(53):325-38.

9. Merhy EE. O cuidado é um acontecimento, e não um ato. Em: Conselho Federal de Psicologia. Fórum Nacional de Psicologia e Saúde Pública: Contribuições técnicas e políticas para avançar o SUS. Brasília: Conselho Federal de Psicologia; 2006. Pp. 69-78.

10. Rauter C, Passos E, Benevides R, organizadores. Clínica e Política: subjetividade e violação de direitos humanos. Rio de Janeiro: TeCorá Editora; 2002.

11. Passos E, Benevides R. A construção do plano da clínica e o conceito de transdisciplinaridade. Psic Teor Pesq. 2000;16(1):71-9. de bolsa de doutorado (2011-2015) e à Equipe POP RUA (Consultório na Rua Rio de Janeiro, Centro, 2010-2013).

Conflitos de interesse. Nada declarado pelos autores.

Declaração. As opiniões expressas no manuscrito são de responsabilidade exclusiva dos autores e não refletem necessariamente a opinião ou política da RPSP/PAJPH ou da Organização PanAmericana da Saúde (OPAS).

12. Franco TB. Produção do cuidado e produção pedagógica: integração de cenários do sistema de saúde no Brasil. Interface (Botucatu). 2007;11(23):427-38.

13. Brasil, Ministério da saúde. Portaria 4 279/2010. Disponível em: http://www. saude.mg.gov.br/index.php?option $=\mathrm{com}$ gmg\&controller $=$ document $\& \mathrm{id}=7578$ Acessado em setembro de 2018.

14. Mendes EV. As redes de atenção à saúde. Cienc Saude Coletiva. 2010;15(5): 2297-305.

15. Merhy EE, Feuerwecker L, Gomes MP. Da repetição à diferença: construindo sentidos com o outro no mundo do cuidado. Em: Franco TB, organizador. Semiótica, afecção \& cuidado em saúde. São Paulo: Hucitec; 2010. Pp. 60-75.

Manuscrito recebido em 14 de janeiro de 2018. Aceito em versão revisada em 9 de agosto de 2018 
ABSTRACT

Contributions of Street Outreach teams to primary health care and management

Keywords
As part of the Unified Health System (SUS) in Brazil, a Street Outreach Program was created with the goals of delivering primary health care (PHC) and guaranteeing ac-cess to health initiatives for homeless populations in the street environment itself, connecting these populations to other services beyond urgent care and emergency facilit-ies. The Program's scope of action involves, in addition to health care, protecting this population against the risks to which they are exposed combined with an effort to guarantee their rights. In this sense, the Street Outreach Clinics strive to ensure equity and access for people with no fixed address, within a system that essentially relies on geographical catchment areas to provide health care. Thus, the establishment of Street Outreach Clinics has introduced new modes of providing health care, and consequently new modes of managing work processes. Based on this articulation between care and management, the present article discusses three levels of intervention for the work of Street Outreach Clinics and teams (the street itself, the health care unit, and institutional networks ), as well as the relationship between this Program and other PHC services and its contribution to reconciling $\mathrm{PHC}$ with its essential attributes, beyond catchment areas.

Health management; universal access to health care services; primary health care; homeless persons; Brazil.
RESUMEN

Contribuciones de los equipos de Consultorio en la Calle para el cuidado y la gestión de la atención básica

Palabras clave
Como parte del Sistema Único de Salud en Brasil, los Consultorios en la Calle y sus equipos fueron creados teniendo como función prioritaria prestar cuidados primarios y garantizar el acceso a las acciones y servicios de salud para poblaciones en situación de calle, en el propio ambiente de la calle, creando vínculos en esa población con otros servicios que no sean solamente los de urgencia o emergencia. Su alcance involucra, además de la atención, la protección contra los riesgos a que está expuesta esa población, combinada con la búsqueda de la garantía de sus derechos. En ese sentido, los Consultorios en la Calle buscan hacer efectiva la equidad y el acceso a las acciones y servicios de salud para una población sin domicilio fijo dentro de un sistema basado esencialmente en la adscripción territorial de la población. Así, la creación del Consultorio en la Calle inaugura nuevos modos de cuidados de la salud y, en consecuencia, nuevos modos de gestionar el proceso de trabajo. A partir de esa articulación entre cuidado y gestión, el presente artículo discute tres planos de intervención donde se da la práctica de los equipos de Consultorio en la Calle (la propia calle, la sede o unidad de referencia y las redes institucionales), su relación con los demás servicios de atención primaria de salud y su contribución para reconciliar la atención primaria de la salud con sus atributos fundamentales, además de la adscripción del territorio geográfico.

Gestión en salud; acceso universal a los servicios de salud; atención primaria de salud; personas sin hogar; Brasil. 